\title{
Time-Space \& Space-Time Elements for Unsteady Advection-Dominated Problems
}

\author{
Maria Isabel Asensio ${ }^{1}$, Blanca Ayuso ${ }^{2}$ and Giancarlo Sangalli ${ }^{2}$ \\ 1 Departamento de Matemática Aplicada, Plaza de la Merced s/n, Universidad de \\ Salamanca, Salamanca 37008, Spain \\ mas@usal.es \\ 2 Istituto di Matematica Applicata e Tecnologie Informatiche CNR, Via Ferrata 1, \\ Pavia 27100, Italy \\ blanca@imati.cnr.it, sangalli@imati.cnr.it
}

Summary. We present some stabilized methods for a nonstationary advectiondiffusion problem. The methods are designed by combining of some stabilized finite element methods and Discontinuous Galerkin time integration. Numerical experiments are presented comparing the new schemes with the space time elements of [3].

\section{Introduction}

The numerical simulation of advection-diffusion problems has been a subject of active research during the last thirty years. In this paper we look at the unsteady problem. Following with the research initiated in [4], our aim is to study the issue of how some of the stabilization techniques proposed for the steady problem could be appropriately combined and used with time integration Discontinuous Galerkin (DG) methods, so that the resulting fully discretized scheme is able to capture and reproduce the small scales into the coarse ones. Our starting point is based on the simple observation that in the non-stationary problem we have two types of partial differentiation which might be considered of different nature: the spatial convection-diffusionreaction operator and the time derivative which determines the evolution of the convection-diffusion-reaction processes. Therefore, at the very first step of designing the numerical method, two rather different strategies arise:

- discretize at first in time by using a DG method and then apply a stabilized method to approximate the resulting family of stationary problems;

- discretize first in space by means of a stabilized finite element method and then use a DG scheme to integrate the corresponding system of ODE's.

The resulting methods from these two approaches will be described and further compared with the "classical" space-time elements introduced in the 80's by Johnson, Nävert and Pitkäranta in [3]. 
The outline of the paper is as follows. In Sect. 2 we review the stabilization techniques proposed for the stationary problem, that will be further considered. In Sect. 3 we revise the time DG integration and introduce the stabilized methods for the time-dependent problem. Numerical experiments are presented in Sect. 4. For the sake of simplicity we restrict ourselves to the one dimensional problem.

\section{Stabilization techniques for the stationary problem}

Let $\Omega=(0, L)$ and let $f \in L^{2}(\Omega)$ be given. Consider the stationary problem

$$
\mathcal{L} u=-\epsilon u_{x x}+\beta u_{x}+\sigma u=f, \quad \text { in } \Omega, \quad u=0 \quad \text { on } \partial \Omega,
$$

where $\epsilon>0, \sigma \geq 0$ and $\beta$ are assumed to be constants and $\epsilon$ will be typically small. Let $\mathcal{V}=H_{0}^{1}(\Omega)$. The bilinear form associated to $\mathcal{L}$ is defined by

$$
a(u, v)=<\mathcal{L} u, v>\equiv \epsilon \int_{\Omega} u_{x} v_{x} d x+\beta \int_{\Omega} u_{x} v d x+\sigma \int_{\Omega} u v d x, \forall u, v \in \mathcal{V} .
$$

We denote by $\mathcal{L}_{\text {Sym }}$ and $\mathcal{L}_{\text {Skew }}$ the symmetric and skew-symmetric parts of $\mathcal{L}$, respectively. The formal adjoint of $\mathcal{L}$ will be denoted by $\mathcal{L}^{*}=\mathcal{L}_{\text {Sym }}-\mathcal{L}_{\text {Skew }}$.

Let $\mathcal{T}_{h}$ be a partition of $\Omega$ into elements (subintervals) $K$ and let $V_{h} \subset \mathcal{V}$ be the corresponding finite element space of piecewise linear polynomials. The standard Galerkin (SG) approximation of (1) reads:

$$
\text { Find } u_{h}^{S G} \in V_{h} \quad \text { such that } \quad a\left(u_{h}^{S G}, v_{h}\right)=\left(f, v_{h}\right), \quad \forall v_{h} \in V_{h} .
$$

It is well known that the plain Galerkin method on a uniform grid fails to furnish a satisfactory approximation if the diffusion coefficient $\epsilon$ is small with respect to the advection or/and reaction coefficients and to the mesh size $h$. To cope with these difficulties, we consider the next family of strongly consistent methods, which following [1] can be presented in the unified way

$$
\left\{\begin{array}{l}
\text { Find } u_{h}^{S t b} \in V_{h} \text { such that } \\
a\left(u_{h}^{S t b}, v_{h}\right)+\sum_{K \in \mathcal{T}_{h}} \delta_{K}\left(\mathcal{L} u_{h}^{S t b}, \mathcal{L}_{S k e w} v_{h}+\rho \mathcal{L}_{S y m} v_{h}\right)_{K} \\
\quad=\left(f, v_{h}\right)+\sum_{K \in \mathcal{T}_{h}} \delta_{K}\left(f, \mathcal{L}_{S k e w} v_{h}+\rho \mathcal{L}_{S y m} v_{h}\right)_{K}, \quad \forall v_{h} \in V_{h},
\end{array}\right.
$$

where $\rho=0$ gives the SUPG (Stramline Upwind Petrov Galerkin) method[5]; $\rho=1$ gives the GLS (Galerkin/Least Squares ) method [11]; and $\rho=-1$ gives the DWG (Douglas-Wang Galerkin) method [7]. These schemes require an appropriate tuning of the problem-dependent parameter $\delta_{K}$. A straightforward calculation shows that by taking

$$
\delta_{K}:=\left((2 \sigma)+(2|\beta|) / h+(12 \epsilon) / h^{2}\right)^{-1},
$$

the bilinear form $\mathcal{B}^{\rho}$ defining these methods, 


$$
\mathcal{B}^{\rho}\left(w_{h}, v_{h}\right):=a\left(w_{h}, v_{h}\right)+\sum_{K \in \mathcal{T}_{h}} \delta_{K}\left(\mathcal{L} w_{h}, \mathcal{L}_{S k e w} v_{h}+\rho \mathcal{L}_{S y m} v_{h}\right)_{K},
$$

with $w_{h}, v_{h} \in V_{h}$ is coercive in the norm $\||v|\|^{2}:=(\epsilon+h|\beta|)|v|_{H_{0}^{1}(\Omega)}^{2}+$ $\sigma\|v\|_{L^{2}(\Omega)}^{2}$ in all possible regimes and consequently the methods are stable.

We consider next the Link-Cutting Bubble strategy [4], based on the enrichment of the finite element space $V_{h}$. The idea behind is to augment $V_{h}$ by adding a space of discrete bubbles $V_{B}$, which is constructed element by element on a suitable subgrid. The LCB method can be regarded from two different standpoints. On the one hand, by considering $V_{E}=V_{h} \oplus V_{B}$ as a space of piecewise linear functions on a suitable refined grid, the LCB-approximation reduces to the plain Galerkin method:

Find $u_{E}^{L C B} \in V_{E}$ such that $a\left(u_{E}^{L C B}, v_{E}\right)=\left(f, v_{E}\right), \quad \forall v_{E} \in V_{E}$.

On the other hand, by means of static condensation of the bubble degrees of freedom, one gets the stabilized method: Find $u_{h}^{L C B} \in V_{h}$ s.t.:

$$
a\left(u_{h}^{L C B}, v_{h}\right)+\sum_{K \in \mathcal{T}_{h}}\left(M_{K}\left(f-\mathcal{L} u_{h}^{L C B}\right), \mathcal{L}^{*} v_{h}\right)_{K}=\left(f, v_{h}\right), \quad \forall v_{h} \in V_{h} .
$$

where for each $K \in \mathcal{T}_{h}, M_{K}:\left.L^{2}(K) \rightarrow V_{B}\right|_{K}$ is the solution operator of the local bubble problems: $a\left(u_{B}^{L C B}, v_{B}\right)_{K}=\left.\left(f-\mathcal{L} u_{h}^{L C B}, v_{B}\right)_{K} \forall v_{B} \in V_{B}\right|_{K}$, $a(\cdot, \cdot)_{K}$ and $\left.V_{B}\right|_{K}$ being the restrictions to $K$ of $a(\cdot, \cdot)$ and $V_{B}$, respectively.

\section{Stabilized methods for the non-stationary problem}

Given $f \in L^{2}\left([0, T] ; L^{2}(\Omega)\right)$ and $u_{0} \in L^{2}(\Omega)$, consider the model problem:

$$
\left\{\begin{array}{l}
\frac{\partial}{\partial t} u+\mathcal{L} u=f \quad \text { in } Q=\Omega \times(0, T), \\
u_{\left.\right|_{t=0}}=u_{0} \quad \text { on } \Omega, \quad u=0 \quad \text { on } \partial \Omega \times(0, T) .
\end{array}\right.
$$

We next briefly revise the DG method for the time integration of (8). Then, we shall describe the classical space-time elements and the stabilized methods resulting from the two approaches mentioned in the Introduction.

\subsection{DG-methods for the time integration}

Let $0=t_{0}<t_{1}<\ldots<t_{N}=T$ a subdivision of the time interval $(0, T)$, set $J_{n}=\left(t_{n}, t_{n+1}\right]$ with $k=t_{n+1}-t_{n}$, and introduce the strips $\mathcal{S}_{n}:=$ $\left\{(x, t) \in \Omega \times J_{n}\right\}$, for $n=0, \ldots N-1$. The DG approximation in time to $u$, solution of (8), is sought as a piecewise polynomial of degree at most $q \geq 0$ 
in $t$ on each subinterval $J_{n}$, with coefficients in $\mathcal{V}$, i.e., it belongs to the space $\mathcal{W}^{q}:=\left\{v:[0, T] \vee \mathcal{V} ; v_{J_{n}}=\sum_{j=0}^{q} \psi_{j} t^{j}, \psi_{j} \in \mathcal{V}\right\}$. Note that any $v \in \mathcal{W}^{q}$ is allowed to be discontinuous at the nodes of the partition. Let $(\cdot, \cdot)$ denote the standard $L^{2}$-inner product and for $v, w \in \mathcal{W}^{q}$ we denote by

$$
\begin{array}{ll}
(v, w)^{n}:=\int_{\mathcal{S}_{n}} v w d x d t=\int_{J_{n}}(v, w) d t, & v_{+}(x, t)=\lim _{s \rightarrow 0^{+}} v(x, t+s), \\
\left\langle v, w>^{n}:=\int_{\Omega} v\left(x, t_{n}\right) w\left(x, t_{n}\right) d x=\left(v^{n}, w^{n}\right),\right. & v_{-}(x, t)=\lim _{s \rightarrow 0^{-}} v(x, t+s),
\end{array}
$$

and set $[[v]]_{n}=v_{+}^{n}-v_{-}^{n}$. The DG time-discretization of (8) is obtained by imposing on $\mathcal{S}_{n}$ the initial value at $t=t_{n}$ weakly. Thus, the method reduces to find $U \in \mathcal{W}^{q}$ such that on each $J_{n}$ (for $n=0, \ldots N-1$ ), satisfies

$$
\left(\frac{d U}{d t}+\mathcal{L} U, V\right)^{n}+<U_{+}, V_{+}>^{n}=<U_{-}, V_{+}>^{n}+(f, V)^{n} \quad \forall V \in \mathcal{W}^{q} .
$$

For $q=0$ (i.e., piecewise constants) one has $d U / d t=0$ and $U(t)=U^{n+1}=U_{+}^{n}$ in $J_{n}$, so that the method reduces to the modified backward Euler:

$$
a\left(U^{n+1}, \psi\right)+\frac{1}{k}\left(U^{n+1}, \psi\right)=\frac{1}{k}\left(U^{n}, \psi\right)+\left(\frac{1}{k} \int_{J_{n}} f d t, \psi\right) \quad \forall \psi \in \mathcal{V} .
$$

For $q=1$, let $U(t)=U_{0}^{n+1}+\frac{\left(t-t_{n}\right)}{k} U_{1}^{n+1}$ on $J_{n}$ so that we have to find $\mathbf{U}^{(n+1)}$ s.t.:

$$
\frac{1}{k}\left(D \cdot \mathbf{U}^{n+1}, \mathbf{V}\right)+C \cdot \mathbf{a}\left(\mathbf{U}^{n+1}, \mathbf{V}\right)=\frac{1}{k}\left(E \cdot \mathbf{U}^{n}, \mathbf{V}\right)+\left(\mathbf{F}^{n+1}, \mathbf{V}\right) \quad \forall \mathbf{V},
$$

where $\mathbf{U}^{n+1}=\left[U_{0}^{n+1}, U_{1}^{n+1}\right]^{T}$ with $U_{0}^{n+1}, U_{1}^{n+1} \in \mathcal{V}, \mathbf{V}=[\psi, \eta]^{T}, \psi, \eta \in \mathcal{V}$ and

$$
D=\left[\begin{array}{ll}
1 & 1 \\
0 & \frac{1}{2}
\end{array}\right] \quad C=\left[\begin{array}{cc}
1 & \frac{1}{2} \\
\frac{1}{2} & \frac{1}{3}
\end{array}\right] \quad E=\left[\begin{array}{ll}
1 & 1 \\
0 & 0
\end{array}\right] \quad \mathbf{F}^{n+1}=\left[\begin{array}{c}
\frac{1}{k} \int_{J_{n}} f(t) d t \\
\frac{1}{k^{2}} \int_{J_{n}}\left(t-t_{n}\right) f(t) d t
\end{array}\right]
$$

With a small abuse of notation, $\mathbf{a}(\cdot, \cdot)$ should be understood as the matrix $\mathbf{a}(\mathbf{U}, \mathbf{V})=\left[\begin{array}{c}a\left(U_{0}, \psi\right), a\left(U_{1}, \psi\right) \\ a\left(U_{0}, \eta\right), a\left(U_{1}, \eta\right)\end{array}\right]$. Similarly, in what follows we shall denote by $\mathcal{L}$ the scalar operator acting component-wise; i.e., $\mathcal{L} \mathbf{U}=\left[\mathcal{L} U_{0}, \mathcal{L} U_{1}\right]^{T}$.

\subsection{Classical Space-Time Elements}

We describe briefly the method introduced in [3]. For each $n$ consider a quasiuniform partition of the strip $\mathcal{S}_{n}$ with elements of size $h>\epsilon$, and let $V_{h}^{n}$ be a FE subspace of $H^{1}\left(\mathcal{S}_{n}\right)$ based on such partition, such that for $v \in V_{h}^{n}$ it holds $v=0$ on $\partial \Omega \times J_{n}$. By applying successively on each strip $S_{n}$ the stabilized methods of (3) and imposing the initial value at $t=t_{n}$ weakly and the boundary conditions strongly, one obtains the following method: given $u_{-}^{0}$ an approximation to the initial data $u_{0}$, for $n=0, \ldots, N-1$ find $u^{n} \in V_{h}^{n}$ 


$$
\begin{aligned}
& \left(u_{t}+\mathcal{L} u, v\right)^{n}+\left(u_{t}+\mathcal{L} u, \hat{\delta} \cdot\left[v_{t}+\beta v_{x}+\rho \mathcal{L}_{\text {sym }} v\right]\right)^{n}+<u_{+}, v_{+}>^{n}= \\
& =\left(f, v+\hat{\delta} \cdot\left[v_{t}+\beta v_{x}+\rho \mathcal{L}_{s y m} v\right]\right)^{n}+<u_{-}, v_{+}>^{n}, \quad \forall v \in V_{h}^{n},
\end{aligned}
$$

where the parameter $\hat{\delta}$ is set to $\bar{C} h$ if $\epsilon<h$ and 0 otherwise.

\subsection{First Approach: DG in Time + Stabilized Method in Space}

To present the fully discretized methods resulting from the first approach, the key point is to observe that on each slab $S_{n}$, the solution $U^{n+1} \in \mathcal{V}$ of the DG in time method (10) (and resp. (11)), might be regarded as the solution of a "steady" convection-diffusion-reaction problem with some "added extra reaction" $\frac{1}{k}$, coming from the time discretization. Thus, by discretizing (10) in space with any of the stabilized methods (3), leads to the problem: for each $n=0, \ldots N-1$, find $u_{h}^{n+1} \in V_{h}$ s.t.

$$
\begin{aligned}
& \sum_{K \in \mathcal{T}_{h}} \widetilde{\delta}_{K}^{0}\left(\frac{\left(u_{h}^{n+1}-u_{h}^{n}\right)}{k}+\mathcal{L} u_{h}^{n+1}-\frac{1}{k} \int_{J_{n}} f d t,\left[\mathcal{L}_{\text {skew }}+\rho \mathcal{L}_{\text {sym }}+\frac{\rho}{k}\right] v_{h}\right)_{K}+ \\
& a\left(u_{h}^{n+1}, v_{h}\right)+\frac{1}{k}\left(u_{h}^{n+1}, v_{h}\right)-\left(\frac{1}{k} \int_{J_{n}} f d t, v_{h}\right)-\frac{1}{k}\left(u_{h}^{n}, v_{h}\right)=0, \forall v_{h} \in V_{h} .
\end{aligned}
$$

Similarly for the discretization (11) $(q=1)$, we get: for $n=0, \ldots, N-1$ find $\mathbf{U}_{h}^{n+1}=\left[U_{0}^{n+1}, U_{1}^{n+1}\right]^{T}$ that satisfies for all $\mathbf{V}_{h}=\left[v_{h}, w_{h}\right]^{T}$ with $v_{h}, w_{h} \in V_{h}$

$$
\begin{aligned}
& \sum_{K \in \mathcal{T}_{h}} \widetilde{\boldsymbol{\delta}}_{K}^{1}\left(\frac{\left(D \mathbf{U}_{h}^{n+1}-E \mathbf{U}_{h}^{n}\right)}{k}+C \mathcal{L} \mathbf{U}_{h}^{n+1}-\mathbf{F}^{n+1},\left[C \mathcal{L}_{\text {skew }}+\rho C \mathcal{L}_{\text {sym }}+\rho \frac{D}{k}\right] \mathbf{V}_{h}\right)_{K} \\
& +C \mathbf{a}\left(\mathbf{U}_{h}^{n+1}, \mathbf{V}_{h}\right)+\frac{D}{k}\left(\mathbf{U}_{h}^{n+1}, \mathbf{V}_{h}\right)-\left(\mathbf{F}^{n+1}, \mathbf{V}_{h}\right)-\frac{1}{k}\left(E \mathbf{U}_{h}^{n}, \mathbf{V}_{h}\right)=0 .
\end{aligned}
$$

where $\mathbf{a}(\cdot, \cdot)$ is defined as in Sect. 3.1. Note that the weighting operators resulting from the stabilization in this approach, $\left(\mathcal{L}_{\text {skew }}+\delta\left[\mathcal{L}_{\text {sym }}+\frac{1}{k_{n}}\right]\right)$ for $q=0$, and $\left(C \mathcal{L}_{\text {skew }}+\rho\left[C \mathcal{L}_{\text {sym }}+\frac{1}{k} D\right]\right)$ for $q=1$, contain a term coming from the time derivative, but it acts as a reaction term. To ensure the stability of the method in all possible regimes, it can be shown that it is enough to take $\widetilde{\delta}_{K}^{0}:=\left((2 \sigma+2 / k)+2|\beta| / h+12 \epsilon / h^{2}\right)^{-1}$ and $\widetilde{\delta}_{K}^{1}:=$ $\left(2 D / k+2 \sigma C+(2|\beta| C) / h+(12 \epsilon C) / h^{2}\right)^{-1}$, for $q=0$ and $q=1$, respectively. In the last case, we have taken into account that (13) is a system.

For the sake of brevity and clarity in the exposition, we only consider the method that results by discretizing (10) in space by means of the LCB strategy. As before, the key observation is that (10) might be regarded as a convection-difussion-reaction stationary problem with the extra reaction $1 / k$. Then, the idea is to define a new bilinear form on each strip $\mathcal{S}_{n}$

$$
\widetilde{a}(w, v)=a(w, v)+\frac{1}{k}(w, v), \quad w, v \in W^{q}
$$


and construct the bubble subgrid, and consequently the bubble space $\widetilde{V}_{B}$, according to this bilinear form rather than (15), the one associated to the stationary problem. Then, for each $n$ one consider either the (SG) approach (6), but with $\widetilde{V}_{E}=V_{h} \oplus \widetilde{V}_{B}$.

\subsection{Second Approach: Stabilized Method in Space + DG in Time}

We first discretize (8) in space by means of the stabilized methods given in Sect. 2. As for the techniques (3), we are lead to the following system of ODE's:

$$
\begin{aligned}
& \frac{d}{d t}\left(u_{h}, v_{h}\right)+a\left(u_{h}, v_{h}\right)+\sum_{K \in \mathcal{T}_{h}} \delta_{K}\left(\frac{\partial u_{h}}{\partial t}+\mathcal{L} u_{h}, \mathcal{L}_{\text {Skew }} v_{h}+\rho \mathcal{L}_{\text {Sym }} v_{h}\right)_{K}= \\
& =\left(f(t), v_{h}\right)+\sum_{K \in \mathcal{T}_{h}} \delta_{K}\left(f(t), \mathcal{L}_{S k e w} v_{h}+\rho \mathcal{L}_{S y m} v_{h}\right)_{K}, \forall v_{h} \in V_{h},
\end{aligned}
$$

where $u_{h}:[0, T] \longrightarrow V_{h}$ and $\delta_{K}$ is taken as in (4). By Integrating (15) with (10), we get for each $n=0, \ldots N-1$

$$
\begin{aligned}
& \sum_{K \in \mathcal{T}_{h}} \delta_{K}\left(\frac{\left(u_{h}^{n+1}-u_{h}^{n}\right)}{k}+\mathcal{L} u_{h}^{n+1}-\frac{1}{k} \int_{J_{n}} f d t,\left[\mathcal{L}_{\text {skew }}+\rho \mathcal{L}_{\text {sym }}\right] v_{h}\right)_{K}+ \\
& a_{h}\left(u_{h}^{n+1}, v_{h}\right)+\frac{1}{k}\left(u_{h}^{n+1}, v_{h}\right)-\left(\frac{1}{k} \int_{J_{n}} f d t, v_{h}\right)-\frac{1}{k}\left(u_{h}^{n}, v_{h}\right)=0, \forall v_{h} \in V_{h} .
\end{aligned}
$$

and upon integration in time of (15) with (11) we have: for $n=0, \ldots, N-1$ find $\mathbf{U}_{h}^{n+1}=\left[U_{0}^{n+1}, U_{1}^{n+1}\right]^{T}$ that satisfies for all $\mathbf{V}_{h}=\left[v_{h}, w_{h}\right]^{T} v_{h}, w_{h} \in V_{h}$

$$
\begin{gathered}
\sum_{K \in \mathcal{T}_{h}} \delta_{K}\left(\frac{\left(D \mathbf{U}_{h}^{n+1}-E \mathbf{U}_{h}^{n}\right)}{k}+C \mathcal{L} \mathbf{U}_{h}^{n+1}-\mathbf{F}^{n+1},\left[\mathcal{L}_{\text {skew }}+\rho \mathcal{L}_{\text {sym }}\right] \mathbf{V}_{h}\right)_{K}+ \\
C \mathbf{a}_{\mathbf{h}}\left(\mathbf{U}_{h}^{n+1}, \mathbf{V}_{h}\right)+\frac{D}{k}\left(\mathbf{U}_{h}^{n+1}, \mathbf{V}_{h}\right)-\left(\mathbf{F}^{n+1}, \mathbf{V}_{h}\right)-\frac{1}{k}\left(E \mathbf{U}_{h}^{n}, \mathbf{V}_{h}\right)=0 .
\end{gathered}
$$

Notice that unlike for methods (12) or (13) no explicit reference to the time integration or time-discretization is contained in the weighting operator for these stabilized methods ${ }^{3}$.

For the method resulting by considering the LCB strategy, one starts by constructing the subgrid for the local bubble space $V_{B}$, according to the steady operator $\mathcal{L}$, i.e. according to the bilinear form $a$ (is done as for the steady problem). Then, the enriched space $V_{E}=V_{h} \oplus V_{B}$ is built and the LCB approximation is defined by the scheme: Find $u_{E}:[0, T] \rightarrow V_{E}$ such that

$$
\frac{d}{d t}\left(u_{E}(t), v_{E}\right)+a\left(u_{E}(t), v_{E}\right)=\left(f, v_{E}\right), \quad \forall v_{E} \in V_{E}
$$

Then, one uses either (10) or (11) for the DG integration in time of system (17), noting that now $\mathcal{V}$ is approximated by the enriched space $V_{E}=V_{h} \oplus V_{B}$.

\footnotetext{
${ }^{3}$ For this reason it is enough to take $\delta_{K}$ as in (4) to ensure the stability of the
} method in all the regimes we will look at; in particular the advection dominated. 


\section{Numerical Experiments}

The next set of experiments is devoted to evaluate the performance of the stabilization methods introduced before. We have considered problem (8) over $\Omega=(0,1)$ and subject to homogeneous Dirichlet boundary conditions. We have set $\epsilon=10^{-6}, \beta=1, \sigma=0$, the final time $T=0.2$ and we assume $f=0$. The inital data is taken as $u_{0}=1$ if $|x-0.3| \leq 0.1$ and is set to zero otherwise. We have taken a uniform partition of $\Omega$ into subintervals of length $h=|\Omega| / N$, with $N=20,40,80,160,320$. For each $h$, every experiment was carried out with different values of the time step $k$ below which the local time discretizations are desired. $k$ is selected so that the Courant-number $C F L=k|\beta| / h=$ $1,1 / 2,1 / 3,1 / 4,1 / 5,1 / 10$. For the three approaches, linear DG integration in time has been used.

To valuate the quality of the approximate solutions obtained by the different methods we have represented them in Fig. 1 at time $t=0.15$. For the methods obtained with the first and second approaches, we have only represented the approximation obtained with DWG (o joined by a continuous line) and LCB (squares joined with a dotted line). For the classical space-time elements the approximations with all the methods in (3) are represented. It can be observed, that while the classical time-space elements reduce almost completely the spurious oscillations in the numerical approximations, the solution appears to be extremely dissipated. Nevertheless, the approximation with the other approaches while not very diffusive still presents spurious oscillations. We next look to the relative errors in $L^{\infty}\left([0, T] ; L^{1}(\Omega)\right)$-norm against $N$, for the three approaches. They are represented in Fig. 1 for $C F L=1 / 3$ and all diagrams are depicted with the same vertical axes to ease the comparation. Among the stabilization techniques of (3) depicted with $-\mathrm{O}-$, no significant differences can be observed. The LCB stabilization is represented by squares joined with a dotted line, and in both the first and second approaches, is the method producing the smallest errors. For the first and second approaches, an almost first order of convergence can be observed while for the spacetime elements the rate of convergence seems to be close to 0.6. Moreover, the first apprach seems to be the most accurate from the error-diagrams. For space time elements, the errors are substantially higher than for the other
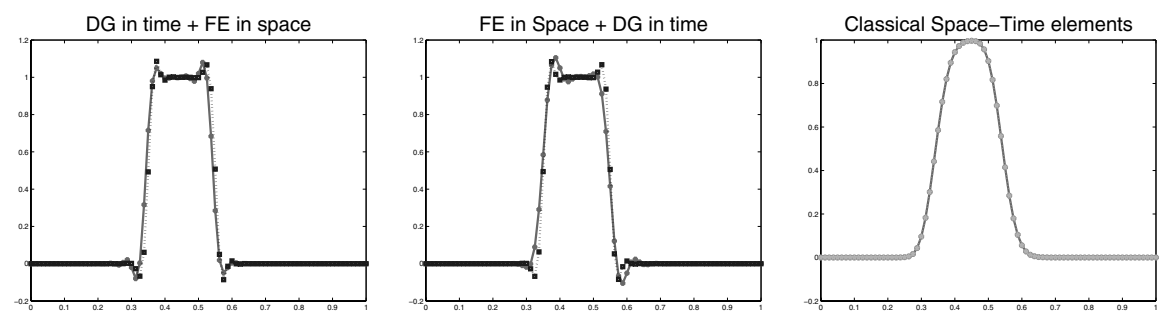

Fig. 1. Approximate solutions with $h=80$ and $C F L=1 / 3$, at time $t=0.15$. 

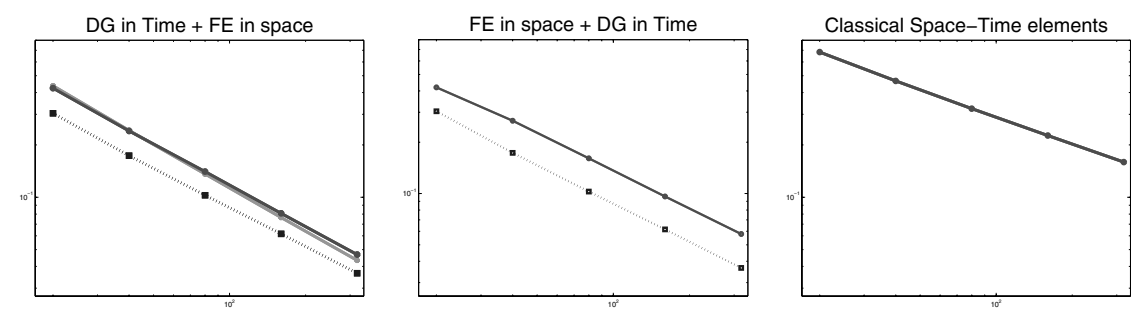

Fig. 2. Convergence Diagrams in $L^{\infty}\left([0, T] ; L^{1}(\Omega)\right)$.

two approaches, possibly due to the amount of dissipation that the scheme introduces.

\section{Acknowledgements}

This research has been partially supported by the Integrated Action ItaloSpanish HI2004-0383 and project HPRN-CT-2002-00286.

\section{References}

1. Baiocchi, C., Brezzi, F.: Stabilization of unstable methods, in: Problemi attuali dell'Analisi e della Fisica Matematica, P.E. Ricci Ed., Universit "La Sapienza", Roma, 59-64 (1993).

2. Brezzi, F., Hauke, G., Marini, L. D. ,Sangalli, G.: Link-cutting bubbles for the stabilization of convection-diffusion-reaction problems. Math. Models Methods Appl. Sci., 13, 445-461 (2003)

3. Johnson, C., Nävert, U., Pitkäranta, J.: Finite element methods for linear hyperbolic problems. Comput. Methods Appl. Mech. Engrg. 45, 285-312 (1984)

4. Asensio, M.I., Ayuso, B., Sangalli, G.: Coupling Stabilized Finite Element methods with Finite Difference time integration for the unsteady advection-diffussionreaction problem, Tech. Report PV-8, (2006)

5. A. N. Brooks, T. J. R. Hughes: Streamline upwind/Petrov-Galerkin formulations for convection dominated flows with particular emphasis on the incompressible Navier-Stokes equations. Comp. Met. Appl. Mech. Engr., 32, 199-259 (1982)

6. Douglas, J., Wang, Jr., Wang, J. P.: An absolutely stabilized finite element method for the Stokes problem. Math. Comp., 52, 495-508 (1989)

7. Hughes, T. J. R., Franca, L. P., Hulbert, G. M.: A new finite element formulation for computational fluid dynamics. VIII. The Galerkin/least-squares method for advective-diffusive equations. Comp. Met. Appl. Mech. Engr., 73, 173-189 (1989) 